\title{
Estudio situacional de la investigación en la formación técnica y tecnológica en el Suroccidente colombiano
}

Balmiro Giraldo Ospina', Victoria Eugenia Rivas ${ }^{2}$

Recibido: 16-03-2016

Aceptado: 09-05-2016

\section{RESUMEN}

El propósito de este artículo es dar a conocer desde la fuente principal, la participación en los programas nacionales de ciencia, tecnología e innovación y el papel que representan las instituciones objeto de estudio, en los planes estratégicos de ciencia, tecnología e innovación de los departamentos de Cauca y Valle del Cauca. La investigación realizada y cuyos resultados se presentan en este escrito, fue desarrollada mediante la técnica de triangulación múltiple, utilizando instrumentos combinados de encuestas, entrevistas y grupos focales al interior de las diferentes instituciones de Educación Superior del Suroccidente colombiano, adscritas a la Mesa Surpacífico de Investigación de la Asociación Colombiana de Instituciones de Educación Superior con Formación Técnica Profesional y/o Formación Tecnológica. Los resultados presentados muestran que en general, la Mesa Surpacífico de Investigación, contribuye con el fortalecimiento de la formación técnica y tecnológica para el desarrollo de actividades en ciencia, tecnología e innovación, para lo cual, se propone un modelo educativo basado en un enfoque curricular investigativo.

Palabras clave: Sistema educativo, desarrollo, formación, ciencia, tecnología, innovación, currículo. 


\title{
Situation on research studies about technical and technological training in southwestern Colombia
}

\begin{abstract}
This article intends to make known, the level of participation in local Science, Technology and Innovation programs nationwide based on the main source as well as the role of the institutions subject of this study, in the strategic plans having to do with Science, technology and innovation in the departments of Cauca and Valle del Cauca. This research project, whose findings are presented in this paper, was conducted by means of a multiple triangulation technique, using mixed instruments such as interviews, surveys and focus groups within the different Higher Education Institutions of southwest Colombia, affiliated to the South Pacific Research Panel of the Colombian Association of Higher Education Institutions with Professional Technical Training and / or Technological Training. These findings demonstrate that the Board contributes in reinforcing technical and technological formation for development in the fields of Science, Technology and Innovation, for which we suggest an educational model based on a Curriculum Research Approach.
\end{abstract}

Key words: Education system, development, education, science, technology, innovation, curriculum.

\section{Introducción}

La sociedad mundial actual se enmarca en una nueva economía de la información y el conocimiento, en donde se distinguen cuatro principales tecnologías, que actualmente están signando las transformaciones del escenario socio-económico internacional: las tecnologías de la información y la comunicación TIC, la nanotecnología, la biotecnología y los nuevos materiales (CEPAL, 2008).

Así mismo, se observa un incremento del desempleo mundial en la fuerza de trabajo con baja capacidad técnica y tecnológica. Además, la tendencia de la economía global de carácter industrial, hacia una economía tercerizada o de servicios (Univalle, 2005), y hacia una reclasificación de 
los sectores económicos, con alto valor tecnológico y cultural, hace que los países empiecen a ser recategorizados por su infraestructura y por su capacidad para apropiarse, generar y usar el conocimiento.

Teniendo en cuenta lo anterior, la educación -principalmente superiordebe propender por la resignificación curricular, hacia la realidad de un entorno globalizado. La preponderancia de los medios electrónicos, ha hecho surgir una nueva cultura de carácter digital, que induce hacia nuevas formas de produciry de trabajar. El desarrollo de las competencias académicas, organizacionales y sociales, prepara a las personas para su inclusión en el mundo contemporáneo, aumentando las posibilidades para su desarrollo personal, laboral y económico.

Así mismo, se considera, que los sistemas educativos nacionales, deben referenciarse desde las necesidades en mano de obra calificada que presentan las pequeñas y medianas empresas, con el fin de facilitar su expansión a través de nuevas inversiones (Cepal, 2013).

También, es necesario articular los diferentes niveles educativos, y homogeneizar las intenciones educativas de las diferentes instituciones educativas públicas y privadas. Por ello, es necesario identificar las necesidades del país, en cuanto a la productividad y competitividad, para lograr sinergias entre el Estado, el sector real de la economía y la academia, con el fin de formar el talento humano, con las competencias pertinentes, hacia un verdadero desarrollo, en donde el crecimiento económico permita la inclusión social y la satisfacción de las necesidades básicas de la población colombiana.

En el ámbito regional, el departamento del Valle del Cauca y sus principales ciudades, presentan perspectivas positivas para su desarrollo. La gobernación del Valle del Cauca, cuenta con el Plan Maestro Integral y de Desarrollo Sostenible del Valle del Cauca 2003-2015, y con la Agenda Regional Prospectiva de Ciencia, Tecnología e Innovación del Valle del Cauca.

En el marco de la política nacional de ciencia, tecnología e innovación (Conpes 3582), se ha elaborado el Plan Estratégico Regional de Ciencia, Tecnología e Innovación del Valle (PERCTI), en donde se resalta la necesidad de fortalecer acciones multidisciplinares en sinergia, para explotar las ventajas competitivas de la región, fortaleciendo el talento humano y fomentando la innovación en el aparato productivo, tendientes 
al desarrollo de las diferentes cadenas productivas identificadas en el valle.

Así mismo, el departamento de Cauca, cuenta con el Plan Estratégico Departamental de Ciencia, Tecnología e Innovación PEDCTI, ratificando la necesidad de consolidar una nueva política nacional de CTI, implementada desde las regiones, con un ejercicio participativo de los diferentes actores y fortalecida a través del surgimiento de diferentes redes del conocimiento. Igualmente, el proyecto "Visión Cauca 2020" es un ejercicio prospectivo de planificación territorial y se ha convertido en la base para construcción del Sistema Regional de Ciencia, Tecnología e Innovación del Departamento de Cauca.

\section{Retos para las instituciones educativas técnicas y tecnológicas}

Según lo expresado en el Plan Estratégico Regional de Ciencia, Tecnología e Innovación del Valle del Cauca PERCTI (2011), el departamento ha hecho importantes avances en términos de oferta variada de programas de formación, a través de un número significativo de universidades e IES, tanto en pregrado como posgrado. Igualmente, en el documento se resalta el progreso en materia de conformación de grupos de investigación adscritos a Colciencias. Sin embargo, la oferta educativa es heterogénea en términos de calidad y se evidencia la concentración en algunas profesiones, además de un bajo nivel de incorporación en CTI (p. 271).

La propuesta desde el PERCTI (2011) va dirigida hacia el "desarrollo de capacidades en ciencia tecnología e innovación regional en la educación media, técnica, tecnológica y superior, orientado a fortalecer la investigación y la innovación pedagógica, curricular e institucional y la articulación de la CTI a los diferentes niveles y a los procesos de formación y a colocar al sistema educativo regional a la altura de los retos del contexto y del propósito del desarrollo integral de la región y del logro de una sociedad del conocimiento y la innovación" (p. 273).

De acuerdo con Tarondeau (1998) para el logro de este tipo de objetivos, es necesario estimular el desarrollo de comunidades de aprendizaje, 
consolidar la organización transversal y canalizar la capitalización de los saberes y los recursos de conocimiento para contribuir al mejoramiento del valor propuesto por las ofertas educativas actuales.

Con referencia a las "comunidades de aprendizaje" Elboj (2003) las define como "uno de estos proyectos caracterizado y basado en las mejores prácticas educativas que están dando respuesta a las necesidades de la sociedad del conocimiento".

Para las IES, esto significa fortalecer y enfocar el pensamiento estratégico de la alta dirección, canalizando esfuerzos de manera participativa con sujetos capaces de gestionar procesos y de manejar tecnologías claves, con el fin de lograr una transformación organizacional que permita ampliar efectivamente las brechas de conocimiento. Con el fin de lograr relaciones efectivas y provechosas entre la universidad y la sociedad, orientadas por el aprendizaje activo, constructivo y significativo, es necesario que las instituciones entiendan el papel articulador del conocimiento, permitiendo la consolidación de cadenas educativas cada vez más largas y continuas (Angulo y Toro, 2001).

Para ello, las IES con formación Técnica y Tecnológica, deben identificar las necesidades del sector productivo en materia de formación y cualificación de su talento humano, que permita incluirlos en los diferentes planes de desarrollo organizacionales, en coherencia con los diferentes planes de desarrollo regionales. Es la oportunidad para liderar procesos de cambio y formular diferentes proyectos interinstitucionales, encaminados a lograr la transformación de su realidad circundante que permita un desarrollo a escala humana y el mejoramiento de la calidad de vida de los habitantes de la región vallecaucana.

Lo anterior se enmarca en el campo de la investigación-acción (Lewin, 1946), que entra en congruencia con una de las propuestas enunciadas en el PERCTI (2011): "Evaluación, seguimiento y diseño de modelos y propuestas de articulación de la formación con entornos tecnoproductivos (urbanos, rurales etc.) e institucionales (I. Educativas, Centros de investigación y desarrollo tecnológico sectorial) y las vocaciones y perspectivas subregionales" (p. 272).

El Consejo Nacional de Educación Superior CESU (2014) presentó al país el documento "Acuerdo por lo Superior 2034: propuesta de Política Pública para la excelencia de la educación superior en Colombia, en el 
escenario de la Paz", con el cual se establecen lo que requiere el país en esta materia para las próximas dos décadas.

Con relación a la investigación (ciencia, tecnología e innovación) el documento establece que las IES son fundamentales para el funcionamiento del Sistema Nacional de Ciencia Tecnología e innovación SNCTel. Así mismo, evidencia la necesidad de reformarlas estructuralmente para que participen activamente en la solución de los problemas del país. La educación terciaria pertinente, rica en conocimientos, ha sido el principal factor orientador del sector educativo para la formulación de políticas públicas y de estrategias institucionales, con el fin de captar y retener el mayor número posible de estudiantes.

La actividad académica universitaria se caracteriza principalmente por sus procesos implícitos didácticos y pedagógicos, transversalizados por la investigación y complementados por la responsabilidad social universitaria, enfocándose hacia el cumplimiento de su misión, para lograr intercambios y colaboraciones que evidencien la flexibilidad curricular, en beneficio de la comunidad académica en general.

En este sentido, las IES son autónomas al ejercer su misión, lo cual, puede lograrse a partir de la caracterización en su Proyecto Educativo Institucional como una institución profesionalizante o de "hacer mayor o menor énfasis en generar conocimiento a partir de procesos de investigación..." (CESU, 2014).

El documento presentado por el CESU, evidencia un análisis prospectivo de la situación del sector educativo en Colombia. En primera instancia, se presenta argumentos suficientes para la reelaboración de una política pública pertinente con la situación actual del país. Luego, después de elaborar un exhaustivo análisis de la situación problémica, se presentan 10 grandes categorías de "problemas nodales", para posteriormente proponer acciones encaminadas al mejoramiento de la Educación Superior enfocada hacia un solo sistema de "educación terciaria".

Entre los principales problemas nodales identificados -relacionados directamente con la investigación como función sustantiva universitariaEI CESU (2014) reconoce "La falta de coherencia y coordinación entre los actores del SCTI, que en ocasiones implica el disímil uso de lenguajes y la carencia de criterios comunes para fomentar y evaluar la investigación" (p. 102). 
Además, en cuanto al propósito de lograr la articulación de las diferentes modalidades y niveles educativos para consolidar un sistema educativo pertinente con la realidad económica y social del país, adolece de insuficiencias fundamentales, iniciando por una clara conceptualización, así se evidencia en el apartado sobre este problema identificado:

La carencia en el país de una conceptualización sobre el alcance y los objetivos de la educación terciaria, las competencias de las diferentes categorizaciones (técnico laboral, técnico profesional, tecnológico). Asociado a lo anterior, no existe claridad sobre el rol que deberían cumplir las diferentes instituciones y actores, sus mecanismos de articulación y de aseguramiento de la calidad (p. 110).

El siguiente compendio obedece a la clasificación de los lineamientos relacionados con la investigación- propuestos por el CESU (2014) con el fin de dar cumplimiento a la visión formulada. Entre otros, ha sido posible identificar los siguientes:

a. Fomentar la interacción de las IES con la empresa impulsando, entre otros, la creación de nuevas spin off académicas. Fortalecer las spin off existentes por parte de las IES que en su Misión y Proyecto Educativo Institucional consideren la innovación como una necesidad (p. 134).

b. Se debe propender porque las IES conformen nodos de conocimiento regional entre los investigadores, grupos, redes y centros de investigación de las diferentes áreas del conocimiento. Ello con el propósito de que en el mediano y largo plazo se institucionalicen estas formas de coordinación y cooperación, capaces de proporcionar la estabilidad y continuidad a los proyectos de investigación de largo aliento (p. 134).

c. Afianzar en los programas de educación superior estrategias pedagógicas que estimulen, a través de componentes transversales del currículo, competencias para el desarrollo de actividades de investigación (p. 135).

d. Establecer, con el concurso del MEN, el CNA, CONACES y COLCIENCIAS, estándares diferenciales de calidad exigibles en investigación, según la tipología de IES que se establezcan y el nivel de programas (técnico, tecnológico, profesional, maestrías 
y doctorados) de que se trate. Recomendar que cada Programa Nacional de Ciencia y Tecnología, en coordinación con las academias, colegios y asociaciones profesionales, determine criterios y parámetros para evaluar el impacto de la investigación por área de conocimiento, para proveer información certera y oportuna a la sociedad, a la industria y a los demás países sobre el tema (p. 135).

e. Institucionalizar el acceso abierto a los resultados de investigación como una de las estrategias principales para que la sociedad se interese, comprenda, valide y utilice el conocimiento. Como canales disponibles para la difusión, con acceso a texto completo o restringido, según criterio de las IES, se publicarán sus resultados de investigación en un Portal Nacional de Publicaciones Científicas Colombianas o en repositorios compartidos (p. 135).

Bajo esta prospectiva, solo falta aunar esfuerzos tendientes a dar cumplimiento a este propósito nacional, enunciado en el "Acuerdo por lo superior 2034" como fundamento del establecimiento de una política pública de Estado en educación, pertinente con la realidad social y económica del país.

El estudio situacional de la investigación es una actividad necesaria para identificar los factores que inciden en las prácticas investigativas de las Instituciones de Educación Superior IES que conforman la Mesa Surpacífico de Investigación adscrita a $\mathrm{ACIET}^{3}$, en relación con el Sistema Nacional de Competitividad de Colombia.

El objetivo del trabajo es caracterizar la participación de dichas instituciones educativas en los planes regionales de ciencia, tecnología e innovación de los Departamentos de Cauca y Valle del Cauca, con el fin de conocer los avances en materia de investigación de cada una de las IES participantes. Esta identificación se constituye en un principal insumo para la toma de decisiones por parte de las directivas de ACIET, con el fin de consolidar una red de investigación contribuyente al sistema nacional de competitividad colombiano. No se conoce la elaboración de otros estudios relacionados con la temática.

Para lograr este propósito investigativo, se pretende elaborar un estudio en diferentes instituciones de educación superior con formación 
técnica y tecnológica, con el fin de identificar cual es y como ha sido su participación en los Planes Estratégicos de Ciencia, Tecnología e Innovación de los Departamentos de Cauca y Valle del Cauca. En ese sentido, es preciso recopilar y analizar la documentación pertinente, respecto a las propuestas, planes y convenios en ciencia, tecnología e innovación de las IES objeto de estudio y su relación con las agendas regionales prospectivas de CTel del Valle y Cauca.

Los resultados del trabajo investigativo permitirán elaborar una propuesta académica que tenga relación con los factores de Ciencia, Tecnología e Innovación, con el propósito de obtener la validación correspondiente porparte de las instituciones que logren implementarla.

\section{Métodos}

\section{Población y muestra}

Para realizar el estudio, el equipo investigador utilizó la técnica de la triangulación múltiple, obteniendo la información por diversos instrumentos -entrevistas semiestructuradas, grupos focales, análisis documental y conclusiones cuantitativas- de las quince (15) IES adscritas a la Mesa Surpacífico de ACIET. La población objeto de estudio está compuesta por dos universidades, cinco instituciones universitarias, tres tecnológicas y cinco técnicas. De estas instituciones, siete son públicas (cuatro técnicas, dos institución universitaria y una universidad) y ocho son privadas (una técnica, tres instituciones universitarias, tres tecnológicas y una universidad).

\section{Técnicas e instrumentos}

Se realizó una recopilación teórica y temática referente a los procesos emprendidos por las instituciones integrantes de la Mesa Surpacífico de investigación perteneciente a ACIET en el Valle del Cauca, para participar de los Planes Estratégicos Regionales de Ciencia, Tecnología e Innovación del Cauca y Valle del Cauca. Junto con la metodología se realizó una recopilación, análisis e interpretación de los conceptos clave que alimentarían las categorías cualitativas a describir e interpretar y variables cuantitativas de análisis. 
También se implementó un análisis estadístico, obteniendo resultados que brindaron soporte para iniciar el proyecto en su fase monográfica. Estos resultados fueron sustentados con documentos o estudios aportados por las instituciones objeto de estudio de la investigación, esbozando conclusiones y posibles recomendaciones desde el equipo de investigación. Para el efecto se realizaron encuestas a cada una de las instituciones. Las encuestas fueron respondidas por los directores de las unidades investigativas.

Las técnicas cualitativas, como entrevistas semi-estructuradas y grupos focales, que se trabajaron en campo en las diferentes instituciones de la muestra, fueron suficientes para confrontar las categorías de análisis y proponer categorías emergentes. Posteriormente a la entrevista y después de haber obtenido información sobre categorías emergentes se gestionó un grupo focal por cada institución con la participación de docentes, estudiantes y administrativos.

Posteriormente se establecieron categorías que denotaron un tópico en sí mismo, y las subcategorías, que detallaron dicho tópico en microaspectos. Estas categorías o subcategorías fueron apriorísticas, es decir, construidas antes del proceso recopilatorio de la información. Transcurrida la indagación inicial emergieron otras categorías, surgidas con el levantamiento de referenciales significativos, lo que se relaciona con la distinción que establece Elliot cuando diferencia entre "conceptos objetivadores" y "conceptos sensibilizadores", en donde las categorías apriorísticas corresponden a los primeros y las categorías emergentes a los segundos (Elliot, 1990) (Cisterna, 2005, p. 64).

\section{Procedimiento}

Se estableció una categorización de las unidades de análisis y sus correspondientes microindicadores, lo cual se efectuó identificando la transversalidad curricular de la investigación en la oferta académica de las diferentes IES analizadas. Una vez obtenida la información, se procedió a su clasificación tomando como referencia los Programas Nacionales de Ciencia, Tecnología e Innovación PNCTel, definidos en el Sistema Nacional de Competitividad colombiano SNCTel.

Para la identificación de las categorías y subcategorías de análisis, se procedió de la siguiente manera: en primera instancia, se identificaron y clasificaron los diversos componentes y factores en unidades temáticas. 
Esta primera codificación permitió identificar dos criterios para la categorización de las unidades de análisis, con relación a la situación problémica: a) factores de orden curricular (Institucional) y b) factores de relación con el SNCTel.

Seguidamente, en cada unidad de análisis se identificaron sus correspondientes componentes temáticos, con el fin de catalogar las categorías de contenido. A su vez, según sus propiedades, estas categorías fueron desagregadas en sub-categorías, de acuerdo con sus temáticas. Esta primera categorización (codificación abierta) facilitó la identificación de las siguientes subcategorías de análisis:

a) Características curriculares investigativas.

b) Perfil ocupacional y competencias investigativas.

c) Prácticas académicas investigativas.

d) Factor administrativo.

e) Factor operativo.

f) Producción escrita.

g) Realización de eventos académicos.

h) Participación en eventos académicos.

i) Relación con el sector externo.

j) Fomento a la investigación.

k) Relación con la comunidad científica internacional.

La depuración de estas variables temáticas, arrojó como resultado la clasificación en cuatro grandes categorías:
A. Transversalidad de la investigación en el currículo.
B. Sistema institucional de investigación.
C. Producción de la investigación.
D. La investigación en el plan de desarrollo institucional.

Después de obtenida esta categorización, se revisaron nuevamente las variables identificadas, para corroborar su pertinencia con el estudio propuesto. Este procedimiento permitió la identificación de atributos correspondientes a cada una de las categorías y sub-categorías, por lo que se hizo necesario reformular o suprimir algunas de ellas. Posteriormente, cada sub-categoría, fue desagregada en microindicadores, con el fin de facilitar el análisis. El resultado obtenido fue la definición operacional de las categorías y sub-categorías, quedando finalmente la siguiente clasificación categorial: 


\section{A. Transversalidad de la investigación en el currículo.}

A1. Características curriculares investigativas.

A2. Perfil ocupacional y competencias investigativas.

A3. Prácticas académicas investigativas.

\section{B. Sistema institucional de investigación.}

B1. Factor administrativo.

B2. Factor operativo.

\section{Producción de la investigación.}

C1. Producción escrita.

C2. Realización de eventos académicos.

C3. Participación en eventos académicos.

D. La investigación en el plan de desarrollo institucional.

D1. Relación con el sector externo.

D2. Fomento a la investigación.

D3. Relación con la comunidad científica internacional.

El procedimiento descrito permitió la sistematización de la información obtenida durante el proceso de estudio, para su posterior análisis y explicación de los resultados, conclusiones y recomendaciones.

A continuación se muestra la categorización de las variables a utilizar para el análisis de los factores de estudio correspondientes a las estrategias, propuesta y acciones de las instituciones participantes respecto a los planes y programas en CTel.

La siguiente tabla, permite observar la clasificación de las cuatro grandes categorías de análisis identificadas y su desagregación en sub-categorías. A su vez, estas sub-categorías, contienen diferentes microindicadores de observación, que se utilizaron para el estudio propuesto. 
Tabla 1. Categorías de análisis.

\begin{tabular}{|c|c|c|}
\hline $\begin{array}{l}\text { Categorías de } \\
\text { análisis }\end{array}$ & Subcategorias & Microindicadores \\
\hline \multirow{16}{*}{$\begin{array}{l}\text { A. } \\
\text { Transversalidad de } \\
\text { la investigación en el } \\
\text { curriculo }\end{array}$} & \multirow{8}{*}{$\begin{array}{l}\text { A.1. Características } \\
\text { curriculares investigativas }\end{array}$} & A.1.1. Enfoque curricular \\
\hline & & $\begin{array}{l}\text { A.1.2. PEI (Integración de las funciones } \\
\text { sustantivas) }\end{array}$ \\
\hline & & A.1.3. Modelo pedagógico \\
\hline & & A.1.4. Planes de formación ofertados \\
\hline & & A.1.5. Modalidad de los planes de estudio \\
\hline & & A.1.6. Créditos académicos \\
\hline & & $\begin{array}{l}\text { A.1.7. Componente de investigación formativa } \\
\text { en contenidos }\end{array}$ \\
\hline & & A.1.8. Areas de formación investigativa \\
\hline & \multirow{4}{*}{$\begin{array}{l}\text { A.2. Perfil ocupacional y } \\
\text { competencias investigativas }\end{array}$} & $\begin{array}{l}\text { A.2.1. Formación profesional (experiencia en } \\
\text { el sector real) }\end{array}$ \\
\hline & & $\begin{array}{l}\text { A.2.2. Formación académica (estudios } \\
\text { realizados) }\end{array}$ \\
\hline & & $\begin{array}{l}\text { A.2.3. Formación pedagógica (estudios y } \\
\text { experiencia en pedagogía) }\end{array}$ \\
\hline & & $\begin{array}{l}\text { A.2.4. Pertinencia de formación con relación a } \\
\text { la investigación }\end{array}$ \\
\hline & \multirow{4}{*}{$\begin{array}{l}\text { A.3. Prácticas académicas } \\
\text { investigativas }\end{array}$} & $\begin{array}{l}\text { A.3.1. Programación de actividades } \\
\text { académicas investigativas }\end{array}$ \\
\hline & & $\begin{array}{l}\text { A.3.2. Tipologia de actividades investigativas } \\
\text { realizadas }\end{array}$ \\
\hline & & $\begin{array}{l}\text { A.3.3. Secuencia y frecuencia de actividades } \\
\text { realizadas }\end{array}$ \\
\hline & & A.3.4. Evaluación de las actividades realizadas \\
\hline \multirow{9}{*}{$\begin{array}{l}\text { B. } \\
\text { Sistema institucional } \\
\text { de investigación }\end{array}$} & \multirow{5}{*}{ B.1. Factor administrativo } & B.1.1. Componente organizacional \\
\hline & & $\begin{array}{l}\text { B.1.2. Componente legal (políticas y } \\
\text { reglamentos) }\end{array}$ \\
\hline & & $\begin{array}{l}\text { B.1.3. Componente tecnológico (Bases de } \\
\text { datos) }\end{array}$ \\
\hline & & B.1.4. Relación con el SNCTel \\
\hline & & B.1.5. Convocatorias internas y externas \\
\hline & \multirow{4}{*}{ B.2. Factor operativo } & B.2.1. Grupos de investigación \\
\hline & & B.2.2. Semilleros de investigación \\
\hline & & B.2.3. Docentes investigadores \\
\hline & & B.2.4. Estudiantes investigadores \\
\hline
\end{tabular}




\begin{tabular}{|c|c|c|}
\hline $\begin{array}{l}\text { Categorias de } \\
\text { análisis }\end{array}$ & Subcategorias & Microindicadores \\
\hline \multirow{11}{*}{$\begin{array}{l}\text { C. } \\
\text { Producción de } \\
\text { investigación }\end{array}$} & \multirow{4}{*}{ C.1. Producción escrita } & C.1.1. Papers \\
\hline & & C.1.2. Libros \\
\hline & & C.1.3. Revistas de investigación \\
\hline & & C.1.4. Otras publicaciones \\
\hline & \multirow{3}{*}{$\begin{array}{l}\text { C.2. Realización de eventos } \\
\text { académicos }\end{array}$} & C.2.1. Congresos \\
\hline & & C.2.2. Simposios \\
\hline & & C.2.3. Otros eventos \\
\hline & \multirow{4}{*}{$\begin{array}{l}\text { C.3. Participación en eventos } \\
\text { de investigación }\end{array}$} & C.3.1. Congresos \\
\hline & & C.3.2. Simposios \\
\hline & & $\begin{array}{l}\text { C.3.3. Encuentros de semilleros de } \\
\text { investigación }\end{array}$ \\
\hline & & C.3.4. Otros eventos \\
\hline \multirow{8}{*}{$\begin{array}{l}\text { D. } \\
\text { La investigación en } \\
\text { el plan de desarrollo } \\
\text { institucional }\end{array}$} & \multirow{3}{*}{$\begin{array}{l}\text { D.1. Relación con el sector } \\
\text { externo }\end{array}$} & $\begin{array}{l}\text { D.1.1. Proyectos interinstitucionales } \\
\text { (Academia) }\end{array}$ \\
\hline & & $\begin{array}{l}\text { D.1.2. Proyectos interinstitucionales (Sector } \\
\text { real) }\end{array}$ \\
\hline & & $\begin{array}{l}\text { D.1.3. Participación de egresados en } \\
\text { proyectos de investigación }\end{array}$ \\
\hline & \multirow{2}{*}{$\begin{array}{l}\text { D.2. Fomento a la } \\
\text { investigación }\end{array}$} & $\begin{array}{l}\text { D.2.1. Politicas de internacionalización de la } \\
\text { investigación }\end{array}$ \\
\hline & & $\begin{array}{l}\text { D.2.1. Existencia de asignación presupuestal } \\
\text { para la investigación }\end{array}$ \\
\hline & \multirow{3}{*}{$\begin{array}{l}\text { D.3. Relación con la } \\
\text { comunidad científica } \\
\text { internacional }\end{array}$} & $\begin{array}{l}\text { D.3.1. Politicas de internacionalización de la } \\
\text { investigación }\end{array}$ \\
\hline & & $\begin{array}{l}\text { D.3.2. Participación en programas } \\
\text { internacionales de investigación }\end{array}$ \\
\hline & & $\begin{array}{l}\text { D.3.3. Estrategias para la internacionalización } \\
\text { de la investigación }\end{array}$ \\
\hline
\end{tabular}

Fuente. Elaboración propia.

\section{Resultados}

Respecto a la transversalidad de la investigación en el currículo, se conoció que en los proyectos educativos institucionales, predomina la tendencia hacia modelos pedagógicos que privilegian el desarrollo de las competencias y desempeños. En algunos casos se enuncian modelos socio-construccionistas. Prevalece la separación de las funciones sustantivas como la docencia, la proyección social, la investigación y la internacionalización. En la práctica académica, se tiene diferentes modalidades como opción de grado para los estudiantes, pero es escasa la elaboración de trabajos signados por la investigación básica o aplicada. 
En general los proyectos de grado tienden hacia los planes de negocios, proyectos académicos de aplicación de conocimientos adquiridos durante la carrera y los diplomados.

En la mayoría de los programas académicos analizados, existe el componente investigativo, más como una asignatura que como una práctica didáctica y pedagógica consuetudinaria.

En conjunto, las IES que hacen parte de la Mesa Surpacífico de ACIET, ofertan los siguientes programas de formación: 40 técnicos (20\%), 75 tecnológicos (38\%), 58 universitarios $(29 \%)$ y 25 posgrados (13\%). Para un total de 198 programas de formación ofertados. Estos grupos se clasificaron bajo el criterio de la participación en los Programas Nacionales de Ciencia, Tecnología e Innovación PNCTel, definidos recientemente por el Departamento Administrativo de Ciencia, Tecnología e Innovación Colciencias. En la Tabla 2 se muestra la correspondiente clasificación.

Tabla 2. Distribución porcentual de programas ofertados.

\begin{tabular}{|c|c|c|}
\hline Programas ofertados & Número & Porcentaje \\
\hline Técnicos & 40 & $20 \%$ \\
\hline Tecnológicos & 75 & $38 \%$ \\
\hline Universitarios & 58 & $29 \%$ \\
\hline Posgrados & 25 & $13 \%$ \\
\hline TOTAL & 198 & $100 \%$ \\
\hline
\end{tabular}

Fuente. Elaboración propia.

En la tabla 3 se evidencia la participación de 106 programas (54\%) en ciencias sociales, humanas y de la educación, $16(8 \%)$ en calidad de vida y medio ambiente, 13 (7\%) en ciencias agropecuarias, $30(15 \%)$ en tecnologías de la información y comunicación, 25 (13\%) en ingenierías, $4(2 \%)$ en ciencias de la tierra y el espacio, $4(2 \%)$ en salud. No se tiene ofertado ningún programa catalogado en ciencias básicas, y tampoco se evidenció la existencia de algún programa específico de formación del recurso humano para la Investigación y la Innovación, en alguna de las instituciones de la población universo analizada. 
Tabla 3. Distribución de la oferta según Planes de Ciencia y Tecnología.

\begin{tabular}{|l|c|c|}
\hline \multicolumn{1}{|c|}{ Programas ofertados } & Número & Porcentaje \\
\hline Ciencias sociales, humanas y de la educación & 106 & $54 \%$ \\
\hline Calidad de vida y medio ambiente & 16 & $8 \%$ \\
\hline Ciencias agropecuarias & 13 & $7 \%$ \\
\hline Tecnologías de la información y comunicación & 30 & $15 \%$ \\
\hline Ingenieria & 25 & $13 \%$ \\
\hline Ciencias de la tierra y el espacio & 4 & $2 \%$ \\
\hline Salud & 4 & $2 \%$ \\
\hline Ciencias básicas & 0 & $0 \%$ \\
\hline Formación del RH para la investigación y la innovación & 0 & $0 \%$ \\
\hline TOTAL & 198 & $100 \%$ \\
\hline
\end{tabular}

Fuente. Elaboración propia.

En trece de las instituciones se tiene unidad de investigación como área académica y/o administrativa. Una de las instituciones respondió que la función investigativa dependía de la vicerrectoría académica. Una institución, por ser sede alterna, no cuenta con una unidad de investigación independiente, sino que sigue los lineamientos para la investigación, de la sede principal ubicada en la capital del departamento del Valle. Se identificaron 59 grupos de investigación -13 categorizados-y 94 líneas de investigación.

Estas líneas de investigación, también se clasificaron bajo el criterio de su participación en los PNCTel. Esta clasificación se muestra en la Tabla 4.

Tabla 4. Distribución porcentual de programas ofertados.

\begin{tabular}{|l|c|c|}
\hline Programas nacionales de ciencia, tecnología e innovación & Número & $\%$ \\
\hline Ciencias sociales, humanas y de la educación & 46 & $49 \%$ \\
\hline Calidad de vida y medio ambiente & 15 & $16 \%$ \\
\hline Ciencias agropecuarias & 8 & $9 \%$ \\
\hline Tecnologías de la información y la comunicación & 19 & $20 \%$ \\
\hline Ingeniería & 6 & $6 \%$ \\
\hline Ciencias de la tierra y el espacio & 0 & $0 \%$ \\
\hline Salud & 0 & $0 \%$ \\
\hline Ciencias básicas & 0 & $0 \%$ \\
\hline Formación del RH para la investigación y la innovación & 0 & $0 \%$ \\
\hline TOTAL & 94 & $100 \%$ \\
\hline
\end{tabular}

Fuente. Elaboración propia. 
Como se observa, se encontraron líneas de investigación atendiendo cinco de los nueve programas de ciencia, tecnología e innovación. La distribución porcentual es la siguiente: 46 en las ciencias sociales, humanas y educación (49\%), 15 en calidad de vida y medio ambiente $(16 \%), 8$ en las ciencias agropecuarias ( $9 \%), 19$ en las tecnologías de la información y la comunicación (20\%) y 6 en las ingenierías (6\%).

Con respecto a la visibilización de la investigación, se encontraron 17 libros, 9 revistas con diferentes ediciones, 70 artículos científicos y 216 publicaciones institucionales.

Las IES objeto de estudio pertenecen a ACIET (10 en calidad de miembrosy 5 en calidad de invitados especiales) por lo cual se ha conformado la Mesa Surpacífico como red colaborativa de investigación. Entre otras redes de investigación, se cuenta con la afiliación de algunas instituciones a la Red Regional de Semilleros de Investigación RREDSI y a la Red Colombiana de Semilleros de Investigación REDCOLSI. Estas redes realizan encuentros regionales, departamentales, nacionales e internacionales de grupos y semilleros de investigación, a los cuales se asiste frecuentemente con proyectos de investigación realizados por grupos de investigación conformados por profesores y estudiantes.

Todas las instituciones manifestaron haber realizado eventos académicos relacionados con la investigación. Se destaca la realización de los simposios internacionales por parte de ACIET, uno de los cuales fue organizado por la Mesa Surpacífico de Investigación, con la participación activa de todos sus miembros.

Entre los proyectos realizados con otras instituciones y agremiaciones, que han tenido reconocimiento por su alto impacto social y económico para la región, se destacan los siguientes:

1. COTECNOVA (Cartago, Valle del Cauca)

Investigón y Preguntina (estrategia para la conformación de comunidades de aprendizaje)

Café norte y Semillas kamerum

2. INTEP (Roldanillo, Valle del Cauca)

Establecimiento de un núcleo de ganado hartón del Valle en la granja departamental cedeagro. 
Caracterización de las propiedades físicas de los suelos del mini distrito de riego del corregimiento de Cajamarca.

Determinación del método de extracción de pectina a partir de la cáscara de pitahaya amarilla. Proyecto realizado en colaboración con la Universidad de Guanajuato de Méjico.

3. Instituto Técnico Agrícola (Buga, Valle del Cauca)

Alianza Minero Energética con la Universidad de Bolívar

Implementación de un centro de producción certificada de plántulas de guadua.

\section{UNICOMFACAUCA (Popayán, Cauca)}

Proyecto "Café Cultura", realizado en colaboración con la empresa Supracafé Colombia y la Universidad Politécnica de Madrid.

Implementación de un modelo innovador de acceso y permanencia en Educación Técnica Profesional y Tecnológica y Empleabilidad en el Norte del Cauca.

5. Universidad del Valle Sede Tuluá.

Estudio sobre el Impacto de la Violencia Política en Mujeres pertenecientes a Organizaciones del Norte y Centro del Valle del Cauca.

Estudio de las propiedades biológicas antimicrobianas de bixa orellana (achiote) y su aplicación a la industria alimenticia: caso productos cárnicos de pollo.

Respecto a proyectos de investigación realizados con instituciones educativas de otros países, solamente tres de las instituciones pertenecientes a la Mesa Surpacífico, han efectuado trabajos investigativos cuyos resultados han impactado significativamente el entorno regional. Se cuentan entre ellas: 1) El Instituto de Educación Técnica Profesional de Roldanillo, que en colaboración con la Universidad de Guanajuato de México, ha realizado los proyectos relacionados con la extracción de la pectina partir de la cáscara de la pitahaya. 2) La Corporación Universitaria Comfacauca, con el proyecto "Café Cultura", en colaboración con la Universidad Politécnica de Madrid, España, y 3) la Fundación Universitaria Católica, que manifestó haber realizado proyectos de investigación con la Universidad de Guatemala.

La siguiente propuesta corresponde a uno de los objetivos del presente trabajo. Se toma como referencia el Modelo Integrador Didáctico y 
Pedagógico para la Educación Superior MIDPES, con el cuál Giraldo (2014) pretende consolidar los factores instrumentales, funcionales y fundamentales, que intervienen el acto formativo en las instituciones educativas. Al respecto enuncia lo siguiente:

El MIDPES se concibe desde el enfoque sistémico, como una organización curricular integradora, compuesta por tres componentes principales: a) el componente metodológico e instrumental -núcleo- denominado Proyecto Integrador (PI), constituido como el dinamo del sistema, b) el componente funcional y relacional, que permite la congruencia entre las teorías y conceptos, con las prácticas y la apropiación de los saberes, yc) el componente fundamental y reglamentario, que orienta y direcciona el proceso formativo desde el orden social, moral y político (2014, p.28).

El modelo fue propuesto a raíz de la dificultad para operacionalizar la estrategia pedagógica denominada Proyecto Integrador PI al interior de una de las Instituciones que hacen parte de este estudio. Otras instituciones han implementado esta estrategia, pero aún no se ha hecho su evaluación a través del modelo propuesto, con el fin de conocer cuál es el impacto que tiene su aplicación, en las prácticas académicas relacionadas con la investigación por parte de estudiantes, docentes y administrativos.

El propósito del proyecto integrador como estrategia pedagógica es "la formación de personas autónomas, proactivas, innovadoras e investigadoras, con la capacidad suficiente para apropiarse de los saberes recibidos durante su proceso de formación y aplicarlo en beneficio del contexto académico, social y económico inmediato" (Giraldo, 2014).

Con objeto de lograr una mejor conceptualización general del modelo, a continuación se presenta la figura 1 , en donde puede observarse su diseño estructural. 


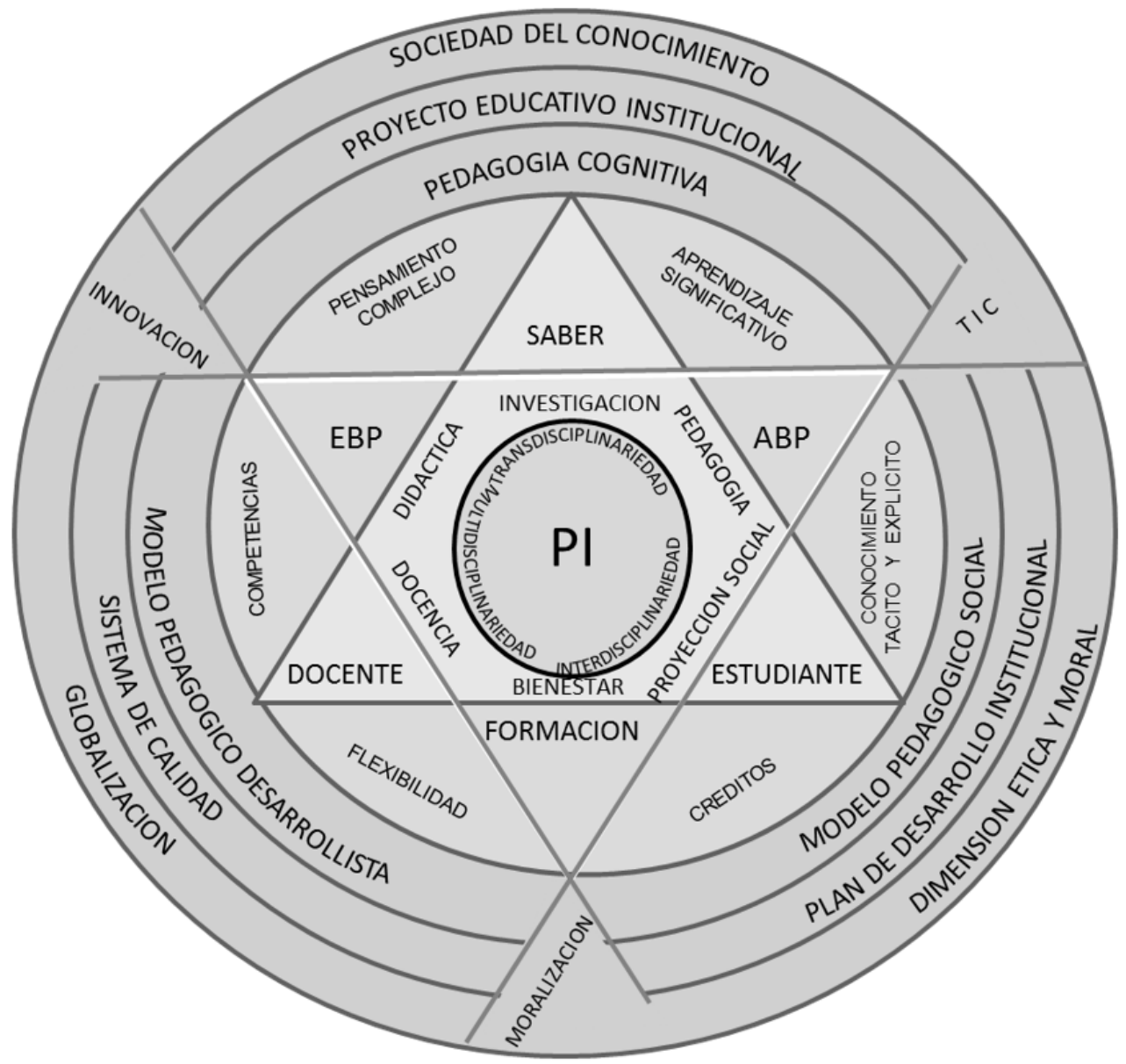

Figura 1. Diseño estructural del MIDPES. Fuente: Giraldo (2014).

Según Giraldo (2014) "El MIDPES se constituye en un arquetipo sistémico, que permite explicar la forma en que se puede generar, apropiar y aplicar el conocimiento en una organización educativa".

El componente instrumental del modelo es el proyecto integrador; el componente relacional y funcional está sustentado en las teorías y conceptos que permite la relación e integración de las funciones sustantivas en la educación superior; y el componente fundamental orienta el quehacer educativo institucional, desde la pedagogía cognitiva y los modelos pedagógicos desarrollista y socioformativo, enfocados desde los paradigmas actuales sociales, morales y políticos relacionados con la educación (Giraldo, 2014). 


\section{Discusión}

Las IES estudiadas cuentan con grupos de investigación, que aunque no todos están reconocidos por Colciencias, han realizado trabajos de investigación que trascienden hacia el impacto social y económico de la región suroccidental de Colombia.

Los trabajos de investigación realizados se consideran de carácter institucional e interinstitucional, en colaboración con organizaciones educativas y/o gremiales. No se conocen trabajos de investigación interinstitucionales por áreas del conocimiento

Solamente tres de las IES han realizado un proyecto cada una, con instituciones de otros países, por lo tanto se considera incipiente la internacionalización de la investigación por parte de la Mesa Surpacífico de ACIET.

Es necesario incentivar la producción intelectual, para ayudar a la visibilización de la investigación y a la categorización de varios grupos de investigación de las IES.

En general, la Mesa Surpacífico de ACIET, está contribuyendo al fortalecimiento de la formación técnica, tecnológica y profesional para el desarrollo de actividades en Ciencia, Tecnología e Innovación, para lo cual, se propone la implementación de un modelo educativo basado en un enfoque curricular investigativo.

Es indispensable que las instituciones educativas con formación técnica y tecnológica lideren un proceso de deconstrucción y reconstrucción curricular, iniciando con la revisión de los contenidos programáticos de las asignaturas desde un enfoque curricular investigativo, fijando políticas claras de apoyo y fomento a las unidades investigativas institucionales.

Con lo indagado en este trabajo, queda un espacio abierto para continuar con futuras investigaciones, que contribuyan y complementen la investigación realizada. Se sugiere la utilización del modelo, con el fin de medir el impacto del modelo integrador didáctico y pedagógico en el mejoramiento de las prácticas pedagógicas universitarias, en especial con la investigación tecnológica. 
Además, es necesario determinar la incidencia que tienen los sistemas educativos institucionales en la formación investigativa de estudiantes y docentes al interior de una institución educativa de educación superior.

Por último, se manifiesta la necesidad de seguir en la sensibilización hacia una cultura de la gestión educativa, con el fin de tratar de disminuir las tensiones generadas entre lo administrativo y lo académico de una IES. Se propone desarrollar la Matriz de Coherencia Institucional que facilite la comprensión sistémica de una institución educativa.

\section{Referencias bibliográficas}

Ángulo, C. y J. R. Toro. (2001). La universidad Académicamente abierta para la actual sociedad del conocimiento. En Orozco, L. E. (comp.), Educación superior. Desafío global y respuesta nacional. Tomo I. Bogotá, Universidad de Los Andes.

CEPAL (2013). Estudio económico de América Latina y el Caribe. Tres décadas de crecimiento desigual e inestable. Santiago de Chile, Naciones Unidas.

Cisterna, C. F. (2005). Categorización y triangulación como procesos de validación del conocimiento en investigación cualitativa. Recuperado el 10 de octubre de 2013, de http://fespinoz.mayo.uson.mx/categorizacion\%20y\%20triangulacion

CODECTI (2011). Plan estratégico Regional de Ciencia, Tecnología e innovación del Valle del Cauca PERCTI, Cali, Gobernación del Valle del Cauca.

CONPES (2008). Política Nacional de Competitividad y Productividad. Documento Conpes 3527, Departamento Nacional de Planeación, Bogotá, Colombia.

CONPES (2009). Política Nacional de Ciencia, Tecnología e Innovación. Documento Conpes 3582, Bogotá, Departamento Nacional de Planeación.

Elboj, C. \& Oliver, E. (2003) Las comunidades de aprendizaje: un modelo de educación dialógica en la sociedad del conocimiento. Huesca. Revista Interuniversitaria de Formación del Profesorado, 17(3): 91-103.

Giraldo B. (2014). Modelo Integrador Didáctico y Pedagógico para la EducaciónSuperior "MIDPES". (Trabajo de grado). Maestría en Educación Superior, Universidad Santiago de Cali. 
OCDE (2005). Manual de Oslo: guía para la recogida e interpretación de datos sobre innovación. Cap. 2, Teoría de la innovación y la necesidad de su medición, Oslo, Unesco.

Tarondeau, J.C. (1998). Le management des savoirs, Colección Que sais-je? № 3407, Presses Universitaires de France, París. Recuperado en mayo 23 de 2014, de http:// www.gobernabilidad.cl/modules.php?name=News\&file=article\&sid

Universidad del Valle (2005). "Bases para el Plan de Desarrollo de la Universidad del Valle 2005-2015", el entorno nacional. Recuperado el 30 de marzo de 2014, de http:// www.univalle.edu.co/plandesarrollo/2analisisentorno.html 